PROCEEDINGS OF THE

AMERICAN MATHEMATICAL SOCIETY

Volume 140, Number 4, April 2012, Pages 1449-1457

S 0002-9939(2011)10972-9

Article electronically published on July 26, 2011

\title{
ACTION OF THE JOHNSON-TORELLI GROUP ON REPRESENTATION VARIETIES
}

\author{
WILLIAM M. GOLDMAN AND EUGENE Z. XIA
}

(Communicated by Bryna Kra)

\begin{abstract}
Let $\Sigma$ be a compact orientable surface with genus $g$ and $n$ boundary components $B=\left(B_{1}, \ldots, B_{n}\right)$. Let $c=\left(c_{1}, \ldots, c_{n}\right) \in[-2,2]^{n}$. Then the mapping class group MCG of $\Sigma$ acts on the relative $\mathrm{SU}(2)$-character variety $\mathfrak{X}_{\mathcal{C}}:=\operatorname{Hom}_{\mathcal{C}}(\pi, \operatorname{SU}(2)) / \mathrm{SU}(2)$, comprising conjugacy classes of representations $\rho$ with $\operatorname{tr}\left(\rho\left(B_{i}\right)\right)=c_{i}$. This action preserves a symplectic structure on the smooth part of $\mathfrak{X}_{\mathcal{C}}$, and the corresponding measure is finite. Suppose $g=1$ and $n=2$. Let $\mathcal{J} \subset$ MCG be the subgroup generated by Dehn twists along null homologous simple loops in $\Sigma$. Then the action of $\mathcal{J}$ on $\mathfrak{X}_{\mathcal{C}}$ is ergodic for almost all $c$.
\end{abstract}

\section{INTRODUCTION}

Let $\Sigma=\Sigma_{g, n}$ be a compact oriented surface of genus $g$ with $n$ boundary components $B=\left\{B_{1}, \ldots, B_{n}\right\}$. Let $\pi=\pi_{1}(\Sigma)$ denote its fundamental group. The mapping class group MCG consists of isotopy classes of orientation-preserving homeomorphisms of $\Sigma$ which pointwise fix each $B_{i}$. Alternatively, MCG is the image under the quotient homomorphism

$$
\operatorname{Aut}(\pi) \longrightarrow \operatorname{Out}(\pi):=\operatorname{Aut}(\pi) / \operatorname{Inn}(\pi)
$$

of the subgroup Aut $(\pi, \mathcal{B})$ of all automorphisms of $\pi$ that preserve the set $\mathcal{B}$ of conjugacy classes of the cyclic subgroups $\pi_{1}\left(B_{i}\right) \subset \pi$ and correspond to orientationpreserving homeomorphisms.

Let $G$ be a Lie group. Then $G$ acts on $\operatorname{Hom}(\pi, G)$ by conjugation. Let

$$
\mathfrak{X}(G)=\operatorname{Hom}(\pi, G) / G \text {. }
$$

Let $\mathcal{C}=\left\{C_{1}, \cdots, C_{n}\right\}$, where $C_{i} \subseteq G$ is a conjugacy class for $1 \leq i \leq n$. Then the relative representation variety is

$$
\operatorname{Hom}_{\mathcal{C}}(\pi, G)=\left\{\rho \in \operatorname{Hom}(\pi, G): \rho\left(B_{j}\right) \in C_{j}, \text { for } 1 \leq j \leq n\right\} .
$$

The group $G$ acts on $\operatorname{Hom}_{\mathcal{C}}(\pi, G)$ by conjugation and the moduli space is the quotient

$$
\mathfrak{X}_{\mathcal{C}}(G)=\operatorname{Hom}_{\mathcal{C}}(\pi, G) / G
$$

Received by the editors April 26, 2010 and, in revised form, December 24, 2010.

2010 Mathematics Subject Classification. Primary 57M05, 22D40, 13P10.

The first author gratefully acknowledges partial support by National Science Foundation grant DMS070781.

The second author gratefully acknowledges partial support by the National Science Council, Taiwan, with grants 96-2115-M-006-002 and 97-2115-M-006-001-MY3.

(C)2011 American Mathematical Society Reverts to public domain 28 years from publication 
The group $\operatorname{Aut}(\pi, \mathcal{B})$ acts on $\pi$, preserving $\mathcal{B}$. Hence it acts on $\operatorname{Hom}_{\mathcal{C}}(\pi, G)$. Furthermore the action descends to a MCG-action on $\mathfrak{X}_{\mathcal{C}}(G)$. The moduli space $\mathfrak{X}_{\mathcal{C}}(G)$ has an invariant dense open subset $\mathfrak{X}_{\mathcal{C}}^{U}(G)$ which is a smooth manifold. This subset has an MCG-invariant symplectic structure $\omega$, hence, a natural smooth MCG-invariant measure $\mu[3,5]$.

Denote by $S$ the set of homotopy classes of simple closed curves on $\Sigma$ and by $J \subseteq S$ the null homologous (in $\mathrm{H}_{1}(\Sigma, \mathbb{Z})$ ) subset. The group MCG is generated by Dehn twists $\tau_{a}$ along simple loops in $S$. Denote by $\mathcal{J} \subseteq$ MCG the subgroup generated by Dehn twists along simple loops in $J$ and by $\mathcal{T} \subseteq$ MCG the subgroup generated by Dehn twists $\tau_{a}$ for $a \in J$ and products $\tau_{a} \tau_{b}^{-1}$, where $a$ and $b$ are disjoint but homologous simple loops in $S$.

When $n \leq 1, \mathcal{T}$ is the Torelli group, i.e. the subgroup of MCG acting trivially on $\mathrm{H}^{1}(\Sigma, \mathbb{Z})[8$. Johnson constructed epimorphisms

$$
\begin{cases}\mathcal{T} \longrightarrow \Lambda^{3} \mathrm{H}^{1}(\Sigma, \mathbb{Z}) / \mathrm{H}^{1}(\Sigma, \mathbb{Z}) & \text { for } n=0 \\ \mathcal{T} \longrightarrow \Lambda^{3} \mathrm{H}^{1}(\Sigma, \mathbb{Z}) & \text { for } n=1\end{cases}
$$

and define the kernels to be $\mathcal{J}$ [7, 6].

For $n>1$, our definition of $\mathcal{T}$ relates to the functorial Torelli group (see [11, 12]). The ergodicity of the MCG-action on $\mathfrak{X}_{\mathcal{C}}(\mathrm{SU}(2))$ was proved in [2, 4]. See [10, 9 ] for similar results when $G$ is a general compact group. Here we prove the following ergodicity result:

Theorem 1.1. Suppose $g=1$ and $n=2$. Then the $\mathcal{J}$-action on $\mathfrak{X}_{\mathcal{C}}(\mathrm{SU}(2))$ is ergodic for generic $C_{1}$ and $C_{2}$.

The moduli space $\mathfrak{X}_{\mathcal{C}}(\mathrm{SU}(2))$ possesses a symplectic structure. The group $\mathcal{J}$ is generated by simple loops described above. The same simple loops also correspond to fundamental group elements. These Dehn twist actions embed into the Hamiltonian vector field flows of the trace functions on these corresponding fundamental group elements. It is then a routine matter to produce a set of such Hamiltonian vector fields, whose flows are locally transitive on an open dense (Zariski) subset $U \subseteq \mathfrak{X}_{\mathcal{C}}(\mathrm{SU}(2))$. However since $\mathfrak{X}_{\mathcal{C}}(\mathrm{SU}(2))$ is a real variety $(\mathrm{SU}(2)<\mathrm{SL}(2, \mathbb{C})$ is a real form), $V=\mathfrak{X}_{\mathcal{C}}(\mathrm{SU}(2)) \backslash U$ is of $\mathbb{R}$-codimension 1 . In other words, $V$ may contain "walls" between components of $U$. To prove ergodicity of the $\mathcal{J}$-action, we analyze the vector fields along $V$ explicitly. This then requires an explicit computation of the symplectic form with the aid of a computer. The inability to carry out these explicit computations for curves of higher genuses and/or with more punctures is the main obstacle in generalizing Theorem 1.1 to these curves.

\section{Trace functions and Hamiltonian flows}

This section summarizes some needed results from [4. Let $X$ be a symplectic manifold and $f: X \rightarrow \mathbb{R}$ a smooth function. Denote by $\mathrm{H}(f)$ the associated Hamiltonian vector field.

Proposition 2.1. Let $X$ be a connected symplectic manifold and let $\mathcal{F}$ be a set of real smooth $\mathbb{R}$-valued functions on $X$ such that at every point $x \in X$, the differentials $d f(x)$, for $f \in \mathcal{F}$, span the cotangent space $T_{x}^{*}(X)$. Then the group $\mathfrak{G}$ generated by the Hamiltonian flows of the vector fields $\mathrm{H}(f)$, for $f \in \mathcal{F}$, acts transitively on $X$.

Proof. The proof is a straightforward application of the implicit function theorem; see Lemma 3.2 in [4]. 
Let $G=\mathrm{SL}(2, \mathbb{C})$ and $\mathcal{C}=\left\{C_{1}, \cdots, C_{n}\right\}$ be a family of conjugacy classes in $G$ such that $C_{i}$ is non-parabolic for each $1 \leq i \leq n$. Let $c=\left(c_{1}, \cdots, c_{n}\right) \in \mathbb{C}^{n}$ such that $c_{i}=\operatorname{tr}(A) \in \mathbb{C}$ for all $A \in C_{i}$. Then the representation variety is equivalently defined as

$$
\operatorname{Hom}_{\mathcal{C}}(\pi, G)=\left\{\rho \in \operatorname{Hom}(\pi, G), \operatorname{tr}\left(\rho\left(B_{j}\right)\right)=c_{j}, \text { for } 1 \leq j \leq n\right\} .
$$

In this setting, if $\alpha \in \pi$ is a homotopy class of based loops, then $\mathrm{t}_{\alpha}$, the trace function of $\alpha$ on $\mathfrak{X}_{\mathcal{C}}$, is defined as

$$
\operatorname{Hom}(\pi, \operatorname{SL}(2, \mathbb{C})) \stackrel{\mathrm{t}_{\alpha}}{\longrightarrow} \mathbb{C} ; \quad \rho \longmapsto \operatorname{tr}(\rho(\alpha)) .
$$

Since the function $\operatorname{SL}(2, \mathbb{C}) \stackrel{\text { tr }}{\rightarrow} \mathbb{C}$ is $\operatorname{lnn}(\pi)$-invariant, $\mathrm{t}_{\alpha}$ defines a function (also denoted by $\left.\mathrm{t}_{\alpha}\right)$ on $\mathfrak{X}_{\mathcal{C}}(\mathrm{SL}(2, \mathbb{C}))$. Furthermore, when $G=\mathrm{SU}(2), c \in I^{n}$, where $I=[-2,2]$.

Proposition 2.2. Let $\alpha$ be a simple separating curve on $\Sigma$ with Dehn twist $\tau_{\alpha}$. Let $\psi: \mathfrak{X}_{\mathcal{C}} \rightarrow \mathbb{R}$ be a measurable function invariant under the cyclic group $\left\langle\left(\tau_{\alpha}\right)_{*}\right\rangle$. Then $\psi$ is almost everywhere invariant under the flow of $\mathrm{H}\left(\mathrm{t}_{\alpha}\right)$.

Proof. See Proposition 5.4 in [4. $\left.\mathfrak{X}_{c}^{U}\right)$.

For the rest of the paper, we shorten $\mathfrak{X}_{\mathcal{C}}(\mathrm{SU}(2))$ (resp. $\left.\mathfrak{X}_{\mathcal{C}}^{U}(\mathrm{SU}(2))\right)$ to $\mathfrak{X}_{c}$ (resp.

\section{ERgodicity}

For $g=0$ and $n=4$ or for $g=1$ and $n=2$, the fundamental group $\pi$ is isomorphic to the free group of three generators

$$
\mathbb{F}_{3}=\left\langle F_{1}, F_{2}, F_{3}, F_{4} \mid \prod F_{i}\right\rangle
$$

where $F_{i}$ corresponds to a simple closed curve on $\Sigma$. By convention, we also use elements in $\pi$ to denote curves they represent on $\Sigma$.

The coordinate ring of $\operatorname{Hom}\left(\mathbb{F}_{3}, \operatorname{SL}(2, \mathbb{C})\right) / \mathrm{SL}(2, \mathbb{C})$ is $\mathbb{C}[K] /(k)$, where

$$
K=\left\{\mathrm{t}_{4}, \mathrm{t}_{1}, \mathrm{t}_{2}, \mathrm{t}_{3}, \mathrm{t}_{12}, \mathrm{t}_{13}, \mathrm{t}_{23}\right\}
$$

with

$$
\mathrm{t}_{i}=\operatorname{tr}\left(\rho\left(F_{i}\right)\right), \quad \mathrm{t}_{i j}=\operatorname{tr}\left(\rho\left(F_{i} F_{j}\right)\right) \text { for } \rho \in \operatorname{Hom}\left(\mathbb{F}_{3}, \operatorname{SL}(2, \mathbb{C})\right)
$$

and

$$
k:=\mathrm{t}_{12}^{2}+\mathrm{t}_{23}^{2}+\mathrm{t}_{13}^{2}+\mathrm{t}_{12} \mathrm{t}_{23} \mathrm{t}_{13}-\mathrm{c}_{12} \mathrm{t}_{12}-\mathrm{c}_{23} \mathrm{t}_{23}-\mathrm{c}_{13} \mathrm{t}_{13}-\mathrm{c}_{0},
$$

where

$$
\begin{aligned}
\mathrm{c}_{12} & :=\mathrm{t}_{1} \mathrm{t}_{2}+\mathrm{t}_{3} \mathrm{t}_{4}, \\
\mathrm{c}_{23} & :=\mathrm{t}_{2} \mathrm{t}_{3}+\mathrm{t}_{1} \mathrm{t}_{4}, \\
\mathrm{c}_{13} & :=\mathrm{t}_{1} \mathrm{t}_{3}+\mathrm{t}_{2} \mathrm{t}_{4}, \\
\mathrm{c}_{0} & :=4-\mathrm{t}_{1}^{2}-\mathrm{t}_{2}^{2}-\mathrm{t}_{3}^{2}-\mathrm{t}_{4}^{2}-\mathrm{t}_{1} \mathrm{t}_{2} \mathrm{t}_{3} \mathrm{t}_{4} .
\end{aligned}
$$

Let

$$
\Omega=d \mathrm{t}_{4} \wedge d \mathrm{t}_{1} \wedge d \mathrm{t}_{2} \wedge d \mathrm{t}_{3} \wedge d \mathrm{t}_{12} \wedge d \mathrm{t}_{13} \wedge d \mathrm{t}_{23}
$$

A priori, we consider elements in $\mathbb{R}[K]$ as functions on $I^{7}$. Then $\mathfrak{X} \subseteq I^{7}$ is a compact component of $\left\{v \in I^{7}: k(v)=0\right\}$. Hence elements in $\mathbb{R}[K]$ also restrict to functions on $\mathfrak{X}$. 
3.1. The 4-holed sphere. Suppose $g=0$ and $n=4$. The boundary components of $\Sigma$ are

$$
B=\left(B_{1}, B_{2}, B_{3}, B_{4}\right) .
$$

The fundamental group $\pi$ is isomorphic to $\mathbb{F}_{3}$ with the isomorphism $B_{i} \mapsto F_{i}$. Let

$$
\mathrm{F}: \mathfrak{X} \longrightarrow I^{4}, \mathrm{~F}(K)=\left(\mathrm{t}_{1}, \mathrm{t}_{2}, \mathrm{t}_{3}, \mathrm{t}_{4}\right) .
$$

Then for $c=\left(c_{1}, c_{2}, c_{3}, c_{4}\right) \in I^{4}, \mathfrak{X}_{c}$ is a compact component of $\mathrm{F}^{-1}(c)$.

The Johnson kernel $\mathcal{J}$ for the 4 -holed torus is trivial as any non-trivial Dehn twist must be along simple curves that separate the four boundary components into pairs. However one may study a different group action as follows: Fix the boundary components into two pairs $\left\{B_{1}, B_{2}\right\}$ and $\left\{B_{3}, B_{4}\right\}$. Let $J^{\prime} \subseteq S$ be the subset containing all the curves separating $\Sigma$ into two pairs of pants containing $\left\{B_{1}, B_{2}\right\}$ and $\left\{B_{3}, B_{4}\right\}$, respectively. Let $\mathcal{J}^{\prime} \subseteq$ MCG be the subgroup generated by Dehn twists along elements in $J^{\prime}$. In this section, we study the $\mathcal{J}^{\prime}$-action on $\mathfrak{X}_{c}^{U}$. This problem is interesting in its own right and instructive in the study of the $\mathcal{J}$-action on $\mathfrak{X}_{c}^{U}$ when $\Sigma$ is the 2-holed torus.

The symplectic bi-vector field relating to $\omega$ is

$$
W=\left(\partial_{\mathrm{t}_{12}} \wedge \partial_{\mathrm{t}_{23}} \wedge \partial_{\mathrm{t}_{13}}\right)(d k) \text {. }
$$

By convention, we use $F_{i}$ to also denote a simple closed curve it represents. The Dehn twist along the simple closed curve $F_{2} F_{3}$ takes the simple closed curve $F_{1} F_{2}$ to a simple closed curve $F_{0}$. Denote by $\tau_{12}, \tau_{0}$ the Dehn twists along $F_{1} F_{2}$ and $F_{0}$, respectively. Let

$$
\Gamma=\left\langle\tau_{12}, \tau_{0}\right\rangle .
$$

Remark 3.1. Both $F_{1} F_{2}$ and $F_{0}$ separate $\Sigma$ into two components each containing $\left\{B_{1}, B_{2}\right\}$ and $\left\{B_{3}, B_{4}\right\}$, respectively. Hence $\Gamma \subseteq \mathcal{J}^{\prime}$.

Let $\mathcal{M}$ be the space of measurable functions $\mathfrak{X} \rightarrow \mathbb{R}$. The trace functions of $F_{1} F_{2}, F_{0}$ are, respectively,

$$
\mathrm{p}_{12}=\mathrm{t}_{12}, \quad \mathrm{p}_{0}=\mathrm{c}_{12}-\mathrm{t}_{23} \mathrm{t}_{13}-\mathrm{t}_{12} .
$$

Let $\mathrm{H}_{i}=W\left(d \mathrm{p}_{i}\right)$ be the Hamiltonian vector field (notice that, to conserve notation, the subscript index $i$ may mean either a number or a pair of numbers). Let

$$
\mathcal{G}_{i}=\left\{g: \mathfrak{X}^{U} \rightarrow \mathfrak{X}^{U} \mid g \text { is a smooth automorphism, } \mathrm{p}_{i} \circ g=\mathrm{p}_{i}, \mathrm{~F} \circ g=\mathrm{F}\right\} .
$$

Let $\mathcal{G}$ be the group generated by $\mathcal{G}_{0} \cup \mathcal{G}_{12}$ and let $\mathfrak{G} \subseteq \mathcal{G}$ be the subgroup generated by the Hamiltonian flows of the $\mathrm{H}_{i}$ 's. It is immediate that $\Gamma \subseteq \mathcal{G}$. Hence $\mathcal{M}^{\mathcal{G}} \subseteq \mathcal{M}^{\Gamma}$. Suppose $f \in \mathcal{M}^{\left\langle\tau_{i}\right\rangle}$. Then by Proposition 2.2

$$
f=f_{i} \circ \mathrm{p}_{i}
$$

for some $f_{i}: \mathbb{R} \rightarrow \mathbb{R}$. Hence $\mathcal{M}^{\left\langle\tau_{i}\right\rangle} \subseteq \mathcal{M}^{\mathcal{G}_{i}}$. Therefore

$$
\mathcal{M}^{\Gamma} \subseteq \mathcal{M}^{\left\langle\tau_{0}\right\rangle} \cap \mathcal{M}^{\left\langle\tau_{12}\right\rangle} \subseteq \mathcal{M}^{\mathcal{G}_{0}} \cap \mathcal{M}^{\mathcal{G}_{12}}=\mathcal{M}^{\mathcal{G}} .
$$

Hence $\mathcal{M}^{\Gamma} \subseteq \mathcal{M}^{\mathcal{G}} \subseteq \mathcal{M}^{\mathfrak{G}}$.

Let $s \in \mathbb{R}[K]$ such that

$$
s \Omega=d \mathrm{t}_{4} \wedge d \mathrm{t}_{1} \wedge d \mathrm{t}_{2} \wedge d \mathrm{t}_{3} \wedge d k \wedge d \mathrm{p}_{0} \wedge d \mathrm{p}_{12} .
$$

A direct calculation shows that

$$
s=2 \mathrm{t}_{23}^{2}-\mathrm{c}_{23} \mathrm{t}_{23}-2 \mathrm{t}_{13}^{2}+\mathrm{c}_{13} \mathrm{t}_{13} .
$$


Then

$$
V=\left\{v \in I^{7}: s(v)=0\right\}
$$

is the dependency locus; i.e. by definition,

Lemma 3.2. The Hamiltonian vector fields $\mathrm{H}_{0}, \mathrm{H}_{12}$ are linearly dependent at $v$ only if $v \in V$.

Remark 3.3. The purpose is to find a sufficient condition for the set $\left\{d \mathrm{p}_{i}\right\}$ to be linearly independent at $v \in \mathfrak{X}_{c}$. The choices of $d \mathrm{t}_{i}, 1 \leq i \leq 4$, and $d k$ are not canonical. They are chosen because the $f_{i}$ 's $(1 \leq i \leq 4)$ are the boundary traces and $k$ is the defining equation of $\mathfrak{X}$; hence, they are constant on $\mathfrak{X}_{c}$.

Lemma 3.4. The vector field $\mathrm{H}_{12}$ is not tangent to $V$ for almost all $c \in I^{4}$.

Proof. Suppose $\mathrm{H}_{12}$ is tangent to $V$. Then $\mathrm{H}_{12}$ is a derivation on the ring of functions on $V \cap \mathfrak{X}$. Hence

$$
\mathrm{H}_{12}(s)=\left(2 \mathrm{t}_{13}+\mathrm{t}_{12} \mathrm{t}_{23}-\mathrm{c}_{13}\right)\left(4 \mathrm{t}_{23}-\mathrm{c}_{23}\right)+\left(-2 \mathrm{t}_{23}-\mathrm{t}_{12} \mathrm{t}_{13}+\mathrm{c}_{23}\right)\left(-4 \mathrm{t}_{13}+\mathrm{c}_{13}\right)
$$

is zero in $\mathbb{R}[K] /(k, s)$; that is, $\mathrm{H}_{12}(s) \in(k, s)$.

We now compute a Gröbner basis for $(k, s) \subseteq \mathbb{R}[K]$. A direct computation shows that the residue of $\mathrm{H}_{12}(s)$ is not zero. This implies that $\mathrm{H}_{12}(s) \notin(k, s)$. This implies that for a generic $c \in I^{4}, \mathrm{H}_{12}$ is not tangent to $V$.

Theorem 3.5. The $\Gamma$-action on $\mathfrak{X}_{c}$ is ergodic for almost every $c \in I^{4}$.

Proof. Suppose $f \in \mathcal{M}^{\Gamma}$. Then $f \in \mathcal{M}^{\mathfrak{G}}$. For almost all $c \in I^{4}$, the set $Q=$ $\mathfrak{X}_{c}^{U} \cap V$ has measure zero and divides $\mathfrak{X}_{c}^{U}$ into a finite number of components. Let $A \subseteq \mathfrak{X}_{c}^{U} \backslash Q$ be a connected component. By Lemma 3.2 the fibres of $\mathrm{p}_{12}, \mathrm{p}_{0}$ are tangent to each other at $v$ only if $v \in Q$. Hence, by Proposition 2.1, $f$ must be constant almost everywhere on $A$.

Lemma 3.4 implies that there is a Zariski dense subset of $Q$ upon which $\mathrm{H}_{12}$ is not tangent to $Q$. Hence there exists a smooth vector field (namely $\mathrm{H}_{12}$ ) that flows across $Q$ between adjacent components. This implies that if $v_{0}, v_{1} \in \mathfrak{X}_{c}^{U} \backslash Q$, then there exists $g \in \mathcal{G}$ such that $g\left(v_{0}\right)=v_{1}$. Since $\mathfrak{X}_{c}^{U}$ is smooth and connected, $f$ is constant almost everywhere on $\mathfrak{X}_{c}^{U} \backslash Q$. Since $\mathfrak{X}_{c}^{U}$ is open and dense in $\mathfrak{X}_{c}$ and $Q$ has measure zero, the theorem follows.

3.2. The 2-holed torus. This case runs in parallel with that of the 4-holed sphere above. Let $g=1$ and $n=2$. The boundary components of $\Sigma$ are $B=\left(B_{1}, B_{2}\right)$. The fundamental group $\pi$ is isomorphic to $\mathbb{F}_{3}$ as described by the ribbon graph in Figure 1, with $B_{1} \mapsto F_{1} F_{2} F_{3}$ and $B_{2} \mapsto F_{1} F_{3} F_{2}$.

The sum and product formulas are, respectively [1]:

$$
\begin{aligned}
& k_{s}=\mathrm{t}_{13} \mathrm{t}_{2}+\mathrm{t}_{1} \mathrm{t}_{23}+\mathrm{t}_{12} \mathrm{t}_{3}-\mathrm{t}_{1} \mathrm{t}_{2} \mathrm{t}_{3}, \\
& k_{p}=\mathrm{t}_{2}^{2}+\mathrm{t}_{3}^{2}+\mathrm{t}_{1}^{2}+\mathrm{t}_{12}^{2}+\mathrm{t}_{13}^{2}+\mathrm{t}_{23}^{2}+\mathrm{t}_{12} \mathrm{t}_{13} \mathrm{t}_{23}-\mathrm{t}_{2} \mathrm{t}_{3} \mathrm{t}_{23}-\mathrm{t}_{2} \mathrm{t}_{1} \mathrm{t}_{12}-\mathrm{t}_{3} \mathrm{t}_{1} \mathrm{t}_{13}-4 .
\end{aligned}
$$

Notice that $k=k_{p}-\mathrm{t}_{4}\left(k_{s}-\mathrm{t}_{4}\right)$. Let

$$
\mathrm{F}: \mathfrak{X} \longrightarrow I^{2}, \quad \mathrm{~F}(K)=\left(\mathrm{t}_{4}, k_{s}-\mathrm{t}_{4}\right) .
$$

Then for $c=\left(c_{1}, c_{2}\right) \in I^{2}, \mathfrak{X}_{c}$ is a compact component of $\mathbf{F}^{-1}(c)$.

Remark 3.6. The moduli space $\mathfrak{X}_{c}$ is the subspace of $\mathfrak{X}$ defined by $k_{s}=c_{1}+c_{2}$ and $k_{p}=c_{1} c_{2}$ 


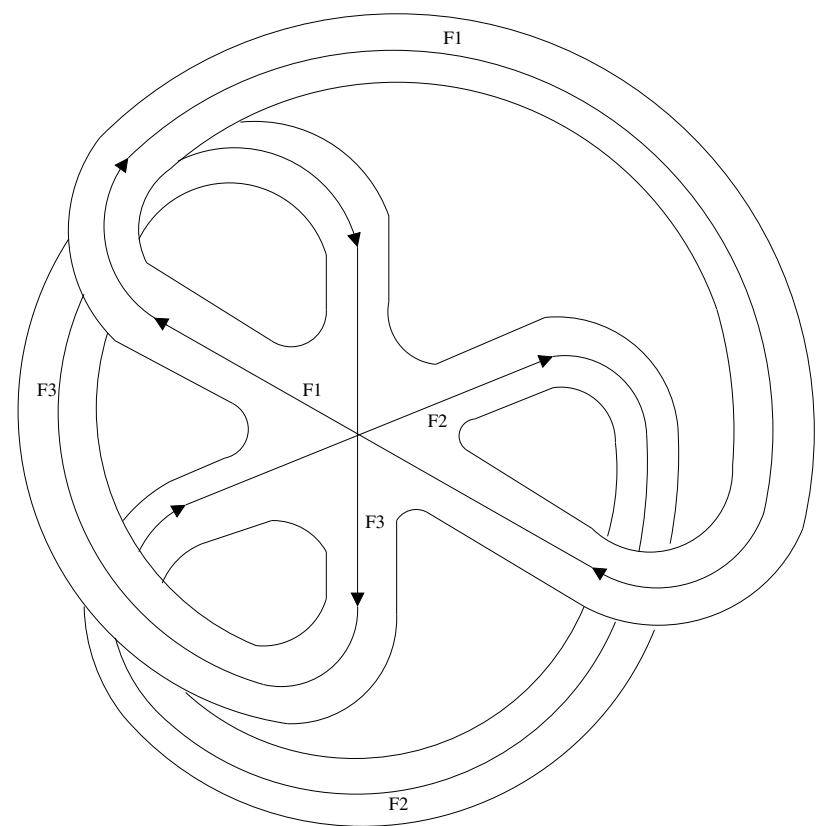

FiguRE 1. A ribbon graph representing a 2-holed torus

With respect to the coordinates $K$, the canonical symplectic bi-vector field $W$ relating to $\omega$ is (this can be computed from the ribbon diagram of Figure 1) the $7 \times 7$ skew-symmetric matrix specified by the following (the unspecified terms are zero; i.e. after filling in these specified terms and making it skew-symmetric, the rest of the terms are zero):

$$
\left\{\begin{array}{l}
W_{2,3}=-2 \mathrm{t}_{12}+\mathrm{t}_{1} \mathrm{t}_{2}, \quad W_{2,4}=2 \mathrm{t}_{13}-\mathrm{t}_{1} \mathrm{t}_{3}, \\
W_{2,5}=-\mathrm{t}_{1} \mathrm{t}_{12}+2 \mathrm{t}_{2}, \quad W_{2,6}=\mathrm{t}_{1} \mathrm{t}_{13}-2 \mathrm{t}_{3}, \\
W_{3,4}=-2 \mathrm{t}_{23}+\mathrm{t}_{2} \mathrm{t}_{3}, \quad W_{3,5}=-2 \mathrm{t}_{1}+\mathrm{t}_{12} \mathrm{t}_{2}, \quad W_{3,7}=-\mathrm{t}_{2} \mathrm{t}_{23}+2 \mathrm{t}_{3}, \\
W_{4,6}=2 \mathrm{t}_{1}-\mathrm{t}_{13} \mathrm{t}_{3}, \quad W_{4,7}=-2 \mathrm{t}_{2}+\mathrm{t}_{23} \mathrm{t}_{3}, \\
W_{5,6}=-\mathrm{t}_{12} \mathrm{t}_{13}-2 \mathrm{t}_{23}+2 \mathrm{t}_{2} \mathrm{t}_{3}, \quad W_{5,7}=2 \mathrm{t}_{13}+\mathrm{t}_{12} \mathrm{t}_{23}-2 \mathrm{t}_{1} \mathrm{t}_{3}, \\
W_{6,7}=-2 \mathrm{t}_{12}+2 \mathrm{t}_{1} \mathrm{t}_{2}-\mathrm{t}_{13} \mathrm{t}_{23} .
\end{array}\right.
$$

The Dehn twist along the simple closed curve $F_{1}$ takes the simple closed curve $F_{2} F_{3} F_{2}^{-1} F_{3}^{-1}$ to a simple closed curve $F_{0}$. Denote by $\tau_{i j}$ the Dehn twists along $F_{i} F_{j} F_{i}^{-1} F_{j}^{-1}$ and by $\tau_{0}$ the Dehn twist along $F_{0}$. Let

$$
\Gamma=\left\langle\tau_{12}, \tau_{23}, \tau_{13}, \tau_{0}\right\rangle
$$

Remark 3.7. $\Gamma \subseteq \mathcal{J}$. 
Let $\mathcal{M}$ be the space of all measurable functions $\mathfrak{X} \rightarrow \mathbb{R}$. The trace functions for various $i$ are

$$
\begin{aligned}
\mathrm{p}_{12}= & -2+\mathrm{t}_{1}^{2}+\mathrm{t}_{12}^{2}-\mathrm{t}_{1} \mathrm{t}_{12} \mathrm{t}_{2}+\mathrm{t}_{2}^{2}, \\
\mathrm{p}_{23}= & -2+\mathrm{t}_{2}^{2}+\mathrm{t}_{23}^{2}-\mathrm{t}_{2} \mathrm{t}_{23} \mathrm{t}_{3}+\mathrm{t}_{3}^{2}, \\
\mathrm{p}_{13}=-2 & +\mathrm{t}_{1}^{2}+\mathrm{t}_{13}^{2}-\mathrm{t}_{1} \mathrm{t}_{13} \mathrm{t}_{3}+\mathrm{t}_{3}^{2}, \\
\mathrm{p}_{0}= & \mathrm{c}_{0}+\mathrm{t}_{1} \mathrm{t}_{12} \mathrm{t}_{2}+\mathrm{t}_{4} \mathrm{t}_{13} \mathrm{t}_{2}+\mathrm{t}_{1} \mathrm{t}_{4} \mathrm{t}_{23} \\
& \quad+\mathrm{t}_{12} \mathrm{t}_{4} \mathrm{t}_{3}-\mathrm{t}_{1} \mathrm{t}_{13} \mathrm{t}_{3}-\mathrm{t}_{1} \mathrm{t}_{12} \mathrm{t}_{23} \mathrm{t}_{3}+\mathrm{t}_{2} \mathrm{t}_{23} \mathrm{t}_{3}+\mathrm{t}_{1}^{2} \mathrm{t}_{3}^{2}-2 .
\end{aligned}
$$

Let $\mathrm{H}_{i}=W\left(d \mathrm{p}_{i}\right)$ (again the subscript $i$ may denote a number or a pair of numbers) be the Hamiltonian vector field. Let

$$
\mathcal{G}_{i}=\left\{g: \mathfrak{X}^{U} \rightarrow \mathfrak{X}^{U} \mid g \text { is a smooth automorphism, } \mathrm{p}_{i} \circ g=\mathrm{p}_{i}, \mathrm{~F} \circ g=\mathrm{F}\right\} .
$$

Let $\mathcal{G}$ be the group generated by $\bigcup_{i} \mathcal{G}_{i}$ and let $\mathfrak{G} \subseteq \mathcal{G}$ be the subgroup generated by the flows of $\mathrm{H}_{i}$ 's. It is immediate that $\Gamma \subseteq \mathcal{G}$. Hence $\mathcal{M}^{\mathcal{G}} \subseteq \mathcal{M}^{\Gamma}$. Suppose $f \in \mathcal{M}^{\left\langle\tau_{i}\right\rangle}$. Then by Proposition 2.2,

$$
f=f_{i} \circ \mathrm{p}_{i}
$$

for some $f_{i}: \mathbb{R} \rightarrow \mathbb{R}$. Hence $\mathcal{M}^{\left\langle\tau_{i}\right\rangle} \subseteq \mathcal{M}^{G_{i}}$. Therefore

$$
\mathcal{M}^{\Gamma} \subseteq \bigcap_{i} \mathcal{M}^{\left\langle\tau_{i}\right\rangle} \subseteq \bigcap_{i} \mathcal{M}^{\mathcal{G}_{i}}=\mathcal{M}^{\mathcal{G}} .
$$

Hence $\mathcal{M}^{\Gamma} \subseteq \mathcal{M}^{\mathcal{G}} \subseteq \mathcal{M}^{\mathfrak{G}}$.

Let $V \subseteq \bar{I}^{7}$ be the subvariety defined by the polynomial $s=0$, where

$$
s \Omega=d \mathrm{t}_{4} \wedge d\left(k_{p}-\mathrm{t}_{4}\right) \wedge d k \bigwedge_{i} d \mathrm{p}_{i} .
$$

By definition, $V$ is the dependency locus:

Lemma 3.8. If the set $\left\{\mathrm{H}_{i}\right\}$ is linearly dependent at $v \in \mathfrak{X}_{c}^{U}$, then $v \in V$.

Remark 3.9. Again in complete parallel with the case of the 4-hole sphere, the choices of $d \mathrm{t}_{4}, d\left(k_{s}-\mathrm{t}_{4}\right), d k$ are not canonical. They are chosen because $\mathrm{t}_{4}, k_{s}-\mathrm{t}_{4}$ are the boundary traces and $k$ is the defining equation of $\mathfrak{X}$; hence, they are constant on $\mathfrak{X}_{c}$.

Lemma 3.10. The vector field $\mathrm{H}_{12}$ is not tangent to $V$ for almost all $c \in I^{2}$.

Proof. Suppose $\mathrm{H}_{12}$ is tangent to $V$. Then $\mathrm{H}_{12}$ is a derivation on the ring of functions on $V \cap \mathfrak{X}$. Hence $\mathrm{H}_{12}(s)=0 \in \mathbb{R}[K] /(k, s)$. This implies that $H_{12}(s) \in$ $(k, s)$.

With the aid of a computer, one may compute a Gröbner basis for $(k, s)$ and show that the residue of $\mathrm{H}_{12}(s)$ is not zero. Hence $\mathrm{H}_{12}(s) \notin(k, s)$. This implies that for a generic $c \in I^{2}, \mathrm{H}_{12}$ is not tangent to $s$.

It so happens that $s=s_{1} s_{2}$ is reducible with two factors. Hence one may compute the Gröbner basis $\left(k, s_{1}\right)$ and $\left(k, s_{2}\right)$ and then compute the residues in each cases.

Proposition 3.11. The $\Gamma$-action on $\mathfrak{X}_{c}^{U}$ is ergodic for almost every $c \in I^{2}$. 
Proof. Suppose $f \in \mathcal{M}^{\Gamma}$. Then $f \in \mathcal{M}^{\mathfrak{G}}$. For almost all $c \in I^{2}$, the set $Q=$ $\mathfrak{X}_{c}^{U} \cap V$ has measure zero and divides $\mathfrak{X}_{c}^{U}$ into a finite number of components. Let $A \subseteq \mathfrak{X}_{c}^{U} \backslash Q$ be a connected component. By Lemma 3.8 and Proposition 2.1 $f$ is constant almost everywhere on $A$.

Lemma 3.10 implies that there is a Zariski dense subset of $Q$ upon which $\mathrm{H}_{12}$ is not tangent to $Q$. Hence there exists a smooth vector field (namely $\mathrm{H}_{12}$ ) in $\mathcal{G}_{12}$ that flows across $Q$ between adjacent components. This implies that if $v_{0}, v_{1} \in \mathfrak{X}_{c}^{U} \backslash Q$, then there exists $g \in \mathcal{G}$ such that $g\left(v_{0}\right)=v_{1}$. Since $\mathfrak{X}_{c}^{U}$ is smooth and connected, $f$ is constant almost everywhere on $\mathfrak{X}_{c}^{U} \backslash Q$. Since $Q$ has measure zero, the theorem follows.

Theorem 1.1 follows as $\Gamma \subseteq \mathcal{J}$ and $\mathfrak{X}_{c}^{U}$ is open and dense in $\mathfrak{X}_{c}$.

Added after posting. We discovered a few misstatements after the article was posted online and we correct them here. Between Remark 3.1 and Lemma 3.2 and between Remark 3.7 and Lemma 3.8 there are passages that begin with "Let

$$
\mathcal{G}_{i}=\left\{g: \mathfrak{X}_{\mathcal{C}}^{U} \rightarrow \mathfrak{X}_{\mathcal{C}}^{U} \mid g \text { is smooth, one-to-one, onto and } \mathrm{p}_{i} \circ g=\mathrm{p}_{i}\right\} "
$$

and end with "Hence $\mathcal{M}^{\Gamma} \subseteq \mathcal{M}^{\mathcal{G}} \subseteq \mathcal{M}^{\mathfrak{G}}$." These two passages should both be replaced with the following passage:

Let $\mathfrak{G}_{i}=\mathfrak{G}\left(\mathrm{p}_{i}\right)$ where $\mathfrak{G}\left(\mathrm{p}_{i}\right)$ is the group generated by the Hamiltonian flow of $\mathrm{H}\left(\mathrm{p}_{i}\right)$. Let $\mathfrak{G}$ be the group generated by $\bigcup_{i} \mathfrak{G}_{i}$. Suppose $f \in \mathcal{M}^{\left\langle\tau_{i}\right\rangle}$. Then by Proposition 2.2, $f \in \mathcal{M}^{\mathfrak{G}_{i}}$. Hence $\mathcal{M}^{\left\langle\tau_{i}\right\rangle} \subseteq \mathcal{M}^{\mathfrak{G}_{i}}$. Therefore

$$
\mathcal{M}^{\Gamma} \subseteq \bigcap_{i} \mathcal{M}^{\left\langle\tau_{i}\right\rangle} \subseteq \bigcap_{i} \mathcal{M}^{\mathfrak{G}_{i}}=\mathcal{M}^{\mathfrak{G}}
$$

\section{REFERENCES}

[1] Goldman, William M., Trace coordinates on Fricke spaces of some simple hyperbolic surfaces. Handbook of Teichmüller theory. Vol. II, 611-684, IRMA Lect. Math. Theor. Phys., 13, Eur. Math. Soc., Zürich, 2009. MR2497777 (2010j:30093)

[2] Goldman, William M., Ergodic theory on moduli spaces. Ann. of Math. (2) 146 (1997), no. 3, 475-507. MR1491446 (99a:58024)

[3] Goldman, William M., The symplectic nature of fundamental groups of surfaces. Adv. in Math. 54 (1984), no. 2, 200-225. MR762512 (86i:32042)

[4] Goldman, William M., Xia, Eugene Z., Ergodicity of mapping class group actions on SU(2)character varieties. Chicago Lectures in Mathematics, University of Chicago Press, Chicago, IL, 1992, 591-608.

[5] Huebschmann, Johannes, Symplectic and Poisson structures of certain moduli spaces. I. Duke Math. J. 80 (1995), no. 3, 737-756. MR.1370113 (97f:58027)

[6] Johnson, Dennis, The structure of the Torelli group. III. The abelianization of $\mathcal{T}$. Topology 24 (1985), no. 2, 127-144. MR793179 (87a:57016)

[7] Johnson, Dennis, The structure of the Torelli group. II. A characterization of the group generated by twists on bounding curves. Topology 24 (1985), no. 2, 113-126. MR793178 (86i:57011)

[8] Johnson, Dennis, The structure of the Torelli group. I. A finite set of generators for $\mathcal{I}$. Ann. of Math. (2) 118 (1983), no. 3, 423-442. MR727699 (85a:57005)

[9] Pickrell, Doug; Xia, Eugene Z., Ergodicity of mapping class group actions on representation varieties. II. Surfaces with boundary. Transform. Groups 8 (2003), no. 4, 397-402. MR2015257 (2004m:57039)

[10] Pickrell, Doug; Xia, Eugene Z., Ergodicity of mapping class group actions on representation varieties. I. Closed surfaces. Comment. Math. Helv. 77 (2002), no. 2, 339-362. MR1915045 (2003i:22025) 
[11] Putman, Andrew, Cutting and pasting in the Torelli group. Geom. Topol. 11 (2007), 829-865. MR2302503 (2008c:57049)

[12] van den Berg, B., On the abelianization of the Torelli group, doctoral dissertation, Universiteit Utrecht (2003).

Department of Mathematics, University of Maryland, College Park, Maryland 20742

E-mail address: wmg@math.umd.edu

Department of Mathematics, National Center for Theoretical Sciences, National Cheng-Kung University, TAinan 701, TAiwan

E-mail address: ezxia@ncku.edu.tw 